INPLASY

PROTOCOL

To cite: Wang et al. Efficacy and safety of moxibustion for benign prostatic hyperplasia: a protocol of systematic review and meta-analysis. Inplasy protocol 2021120021. doi: 10.37766/inplasy2021.12.0021

Received: 03 December 2021

Published: 03 December 2021

Corresponding author: Yuxia Ma

sd15662401851@163.com

Author Affiliation:

Shandong University of Traditional Chinese Medicine, Jinan, Shandong, China.

Support: No. 2020GXRC005.

Review Stage at time of this submission: Preliminary searches.

Conflicts of interest:

None declared.

\section{Efficacy and safety of moxibustion for benign prostatic hyperplasia: a protocol of systematic review and meta-analysis}

Wang, JZ1; Deng, TT2; Sun, $\mathrm{H}^{3}$; Sun, $\mathrm{XL}^{4}$; You, YW5; Wang, Y6; Xun, $Q^{7} ; \mathrm{Ma}, \mathrm{YX}^{8}$.

Review question / Objective: How is the efficacy and safety of moxibustion for benign prostatic hyperplasia compared with conventional treatment?

Information sources: The following electronic databases will be searched regardless of language and publication status: Pubmed, MEDLINE, EMBASE, China Biomedical Database, China National Knowledge Infrastructure, VIP Database and Wanfang Database. Prospero, Clinic Trials.gov and Google Scholar will be used to select systematic reviews or ongoing or completed clinical trials. Meanwhile, papers and bibliographies for inclusion in the trials will also be reviewed.

INPLASY registration number: This protocol was registered with the International Platform of Registered Systematic Review and Meta-Analysis Protocols (INPLASY) on 03 December 2021 and was last updated on 05 December 2021 (registration number INPLASY2021120021).

\section{INTRODUCTION}

Review question / Objective: How is the efficacy and safety of moxibustion for benign prostatic hyperplasia compared with conventional treatment?

Condition being studied: Condition being studied: Benign prostatic hyperplasia (BPH) is a common disease in older male goup, with an increasing incidence along with age. Although the prevalence is only $8 \%$ between the ages of $\mathbf{4 0}$ and 50, it increases to $50 \%$ between the ages of 51 and 60 , and to $83 \%$ by the age of 80 . The clinical manifestations of $\mathrm{BPH}$ are frequent urination, urgency, incomplete dribbling of urine and urinary retention, which are collectively referred to as lower urinary tract symptoms, and these symptoms 
seriously affect patient's sleep, daily social interactions and further lead to depressive symptoms. The main pathological change occurring within the prostate hyperplastic tissue is stromal cell proliferation. Androgens are the most important factor influencing the development of prostatic hyperplasia, but they do not directly cause proliferative changes in prostate cells, but indirectly trigger proliferative changes in prostate tissue cells by stimulating the secretion of relevant growth factors from prostate stroma and epithelial cells. However, there is no clear mechanism for the development of BPH, which may also involve chronic inflammation of the prostate, vitamin D deficiency and other theories. Medication and surgery are common treatments for patients with BPH. initial treatment for BPH is medication, usually 5-a-reductase inhibitors or alphablockers, in addition to other types of therapeutic agents such as M-receptor antagonists and herbal preparations. If pharmacological treatment fails, surgical treatment (transurethral resection of the prostate or open surgery) is used for treatment. For patients with BPH with a prostate volume (PV) of 30 to $80 \mathrm{~mL}$, transurethral resection of the prostate (TURP) is considered the gold standard of treatment and is also indicated for those with a prostate volume $>80 \mathrm{~mL}$. Although this treatment is effective, it is highly invasive to the operator, has a long postoperative recovery time, and patients may experience various complications associated with TURP. Therefore, it is particularly important to choose an alternative therapy with good efficacy and few complications. Moxibustion has been found to have therapeutic effects on a variety of diseases through thermal stimulation. It has been found that moxibustion can, through its warm stimulating effect, cause vasodilatation, enhance blood flow dynamics and lower vascular resistance, thus effectively reducing blood viscosity and improving microcirculatory disorders and blood rheology abnormalities in patients with BPH . To date, there is no evidence of systematic evaluation of moxibustion for BPH. Therefore, in order to obtain convincing evidence for clinical practice, we will systematically review published randomised controlled trials and compare the efficacy and safety of moxibustion with pharmacological/non-pharmacological interventions for BPH.

\section{METHODS}

Participant or population: Male patients with symptomatic prostatic hyperplasia (BPH) will be participants with no restrictions on age, duration, race, disease duration or disease severity. These patients appeared with higher than normal urinary symptom scores including the Boya sky score and the International Prostate Symptom Score (IPSS).

Intervention: The treatment group will include any type of moxibustion therapy such as direct moxibustion, indirect moxibustion, thermosensitive moxibustion, natural moxibustion, medicinal moxibustion as part of the individual treatment or working along with other interventions.

Comparator: The control group will be other types of interventions including medication, surgery and other conventional treatments.

Study designs to be included: The study will consist of a prospective randomised controlled clinical trials (RCTs) of moxibustion in the treatment of $\mathrm{BPH}$, language of publication does not have barrier of blinding or restrictions.

Eligibility criteria: Researches with the following characteristics will be excluded: non-randomised controlled trials; studies with interventions that do not meet the requirements of ayurveda; studies involving patients with non-BPH; case reports, animal studies, reviews, conference papers or reports with incomplete significant data that have not been responded to by the appropriate author.

Information sources: The following electronic databases will be searched regardless of language and publication status: Pubmed, MEDLINE, EMBASE, China Biomedical Database, China National 
Knowledge Infrastructure, VIP Database and Wanfang Database. Prospero, Clinic Trials.gov and Google Scholar will be used to select systematic reviews or ongoing or completed clinical trials. Meanwhile, papers and bibliographies for inclusion in the trials will also be reviewed.

Main outcome(s): The treatment group will include any type of moxibustion therapy such as direct moxibustion, indirect moxibustion, thermosensitive moxibustion, natural moxibustion, medicinal moxibustion as part of the individual treatment or working along with other interventions.

Additional outcome(s): The control group will be other types of interventions including medication, surgery and other conventional treatments.

Quality assessment / Risk of bias analysis: The quality of the included literature was assessed using the risk of bias assessment tool recommended in the Cochrane Systematic Assessor's Handbook 5.1.0. Specific methods included: randomisation methods, allocation concealment, blinding of subjects and investigators, blinding for outcome assessment, completeness of outcome data, selective reporting of results, and other sources of bias, each of which was categorised as low risk of bias, high risk of bias, and unclear.

Strategy of data synthesis: Two evaluators independently screened the literature according to inclusion and exclusion criteria. Abstract and main content of the literature that met the screening criteria will be selected after initial analysis. Final selection will be conducted on the basis of the original text of the selected literature. The 3rd evaluator will come involve when there is disagreement. Extracted information includes first author and year of publication, country, number of patient cases, interventions, follow-up time, and outcome indicators. Authors will be responsible for data extraction and management according to the search strategy, including first author and year of publication, country, number of patients, interventions, follow-up time, and outcome indicators.

Subgroup analysis: If heterogeneity is assessed as significant $\left(I^{2} \geq 50 \%\right)$ and sufficient trials are included, subgroup analyses will be conducted subgroup analyses to explore potential sources of heterogeneity depending on the intervention, control and outcome measure.

Sensitivity analysis: Sensitivity analyses are conducted to explore the impact of methodological quality and sample size on the robustness of the review findings, based on the effects of sample size, methodological quality and missing data. The meta-analysis will be repeated after excluding studies with a high risk of bias and outliers that are numerically distant from the rest of the data. The sensitivity analyses will be reported with the summary tables and the review conclusions will relate to comparisons between the two meta-analysis.

Country(ies) involved: China.

Keywords: Benign prostatic hyperplasia, meta-analysis, moxibustion, protocol, systematic review.

Contributions of each author:

Author 1 - Jiaze Wang - Writing-review \& editing and data curation.

Email: wangjiaze1996@outlook.com

Author 2 - Tingting Deng - Formal analysis and resources.

Email: 2531647328@qq.com

Author 3 - Hao Sun - Methodology and software.

Email: 695604395@qq.com

Author 4 - Xiaolu Sun - Formal analysis.

Email: ycycyc333@126.com

Author 5 - Yuwei You - Methodology.

Email: 1229156810@qq.com

Author 6 - Ying Wang - Methodology.

Email: 2552768176@qq.com

Author 7 - Qi Xun - Software.

Email: 409481838@qq.com

Author 8 - Yuxia Ma - Project administration.

Email: sd15662401851@163.com 